\title{
Langevin equation for the squeezing of light by means of a parametric oscillator
}

\author{
T. W. Marshall \\ Department of Mathematics, University of Manchester, Manchester, United Kingdom \\ E. Santos \\ Departamento de Física Moderna, Universidad de Cantabria, Santander, Spain
}

(Received 16 October 1989)

\begin{abstract}
We show that the Langevin equation for a nonlinear-optical system may be obtained directly from the Heisenberg equation of motion for the annihilation operators, provided a certain linearization procedure is valid. We apply the technique to the parametric oscillator used to generate squeezed light and compare our results to those obtained from Fokker-Planck-type equations. We argue that, only when the Wigner, as opposed to the $P$ or $Q$, representation of quantum optics is used, do we get a correct description of the underlying stochastic process. We show how the linearization procedure may be carried out to describe the operation of the parametric oscillator both below threshold, where a squeezed vacuum state results, and above threshold, where we find a squeezed coherent state. In the region of the threshold a heuristic extension of the method leads to a possible description of the system by means of a nonlinear Langevin equation.
\end{abstract}

There is a standard procedure ${ }^{1}$ used in nonlinear optics to obtain the coupled Langevin equations describing the interaction of two or more modes of the light field. A "reversible" Hamiltonian is first constructed, representing the action of the pumping field and of the nonlinear device. To this is added an "irreversible" Hamiltonian representing the interaction of the radiation modes with the walls of the cavities (that is, the mirrors). The equation of motion for the density matrix is now reduced by eliminating the heat-bath modes; for this step we have to make certain assumptions about the relevant relaxation times. Then we pass to a generalized Fokker-Planck equation for a pseudoprobability density by expanding this density matrix in terms of a suitable set of basis states. The final step, from the "Fokker-Planck" equation to a "Langevin" equation, is a heuristic one, and consists of comparing the quantum process with the classical Brownian motion process, for which the latter rather than the former is the more fundamental equation. This is a highly ambiguous procedure; it is not possible, in all representations of the density matrix, to guarantee that the "probability" density is positive, and even when it is positive quantum theory gives us no criterion for deciding whether, as in the classical case, the distribution is over dispersion-free states, or whether there is some kind of irreducible smoothing to be understood in all of the basis states. Indeed, as we shall see, one obtains different Langevin equations from the commonly used densities obtained with the normally, antinormally, and symmetrically ordered ${ }^{2}$ characteristic functions, known respectively as Glauber $P, Q$ (or positive $P$ ), and Wigner representations.

We therefore propose that it is of interest to model the action of the heat bath in a more classical manner. In this way we pass directly from the Heisenberg to the Langevin equation. The advantages of such a treatment are (a) simplicity, (b) it leads always to genuine (positive) probabilities, and (c) it gives us a criterion for choosing which of the Brownian-motion descriptions obtained from the quantum formalism is correct. In this latter connection we shall show in a subsequent publication that certain autocorrelations of the process are given incorrectly by the Langevin equations derived from the $P$ or $Q$ representations, the correct ones being obtained from the $W$ representation.

The passage from Heisenberg to Langevin equation may be rigorously demonstrated for any system ("linear"), whose Hamiltonian is at most quadratic in the annihilation and creation operators, of the optical and heat-bath modes. We shall presently show how to extend the same treatment to a certain weakly coupled, or quasilinear, system, but we begin by considering the linear case. The following lemma is almost trivial, but nevertheless crucial.

Lemma. The Wigner function of a system whose Hamiltonian is at most quadratic in the annihilation and creation operators evolves according to a deterministic equation of motion.

To prove this lemma, all that is required is the observation that, for such a system, the Wigner-Moyal equation ${ }^{3}$ reduces to the Liouville equation of classical mechanics. In sharp contrast, the evolution equations for the Glauber $P$ or the $Q$ functions contain second derivatives even if the Hamiltonian is quadratic and, therefore, they do not have the form of Liouville equations.

Now let us apply the lemma to a system whose Hamiltonian is $(\hbar=1)$

$$
\begin{aligned}
H= & \omega \widehat{a}^{\dagger} \widehat{a}+\frac{1}{2} f(t) \hat{a}^{\dagger 2}+\frac{1}{2} f^{*}(t) \widehat{a}^{2} \\
& +\sum_{j} \omega_{j} \hat{b}_{j}^{\dagger} \hat{b}_{j}+\sum_{j} \chi_{j}\left(\hat{a}^{\dagger} \hat{b}_{j}^{\dagger}+\widehat{a} \hat{b}_{j}^{\dagger}\right),
\end{aligned}
$$

where $\hat{a}^{\dagger}$ is the creation operator of a "photon," $\hat{b}_{j}^{\dagger}$ the creation operator of a "phonon" in the heat bath, and $\chi_{j}$ 
are coupling constants. Then the Wigner distribution $\mathcal{W}\left(\alpha,\left\{\beta_{j}\right\}\right)$ satisfies a differential equation of the form

$$
\frac{\partial \mathcal{W}}{\partial t}=\mathcal{L} \mathcal{W}
$$

where $\mathcal{L}$ is the Liouville operator, linear in $\partial / \partial \alpha, \partial / \partial \beta_{j}$, and their conjugates. This corresponds to a deterministic time evolution given by

$$
\begin{aligned}
& \dot{\alpha}=-i \omega \alpha-i f(t) \alpha^{*}-i \sum_{j} \chi_{j} \beta_{j}, \\
& \dot{\beta}_{j}=-i \omega_{j} \beta_{j}-i \chi_{j} \alpha .
\end{aligned}
$$

We note that these equations are formally identical with the Heisenberg equation for $\hat{a}$ and $\hat{b}_{j}$, for which there is an established procedure ${ }^{4}$ for eliminating the heat-bath modes, using the Wigner-Weisskopf approximation. We now see that an almost identical procedure may be applied to these "Newtonian" equations. Indeed, a formal integration of the second gives

$$
\begin{aligned}
\beta_{j}(t)= & \beta_{j}(0) \exp \left(-i \omega_{j} t\right) \\
& -i \chi_{j} \int_{0}^{t} \alpha\left(t^{\prime}\right) \exp \left[i \omega_{j}\left(t^{\prime}-t\right)\right] d t^{\prime}
\end{aligned}
$$

and this, when substituted in the first equation, gives

$$
\dot{a}=-i(\omega-i \gamma) a-i f(t) a^{*}+L(t),
$$

where a (small) renormalization of the frequency $\omega$ has been made, the constant $\gamma$ is given by

$$
\gamma=\pi|\chi(\omega)|^{2} \rho(\omega),
$$

$\rho(\omega)$ being the density function of the reservoir oscillators, and $L(t)$ is a (complex) white noise whose mean and variance are given by

$$
\begin{aligned}
& \langle L(t)\rangle=0=\left\langle L(t) L\left(t^{\prime}\right)\right\rangle, \\
& \left\langle L(t) L^{*}\left(t^{\prime}\right)\right\rangle=2 D \delta\left(t-t^{\prime}\right) .
\end{aligned}
$$

The diffusion constant $D$ is essentially the spectral density, at frequency $\omega$, of the heat bath, which is the Planck distribution, including zero-point energy, of "phonons"

$$
2 D=\gamma(1+2 \bar{n})=\gamma \operatorname{coth}(\omega / 2 k T),
$$

where $T$ is the temperature.

A few comments are in order. In the first place, we point out that the elimination of the heat-bath degrees of freedom transforms the deterministic equations of motion (3) into the stochastic differential (Langevin) equation (5). We have changed from $\alpha$ to $a(t)$ in order to emphasize this fact. The solution of (5) is a (Wigner) probability density $W(\alpha, t)$ evolving according to

$$
\begin{aligned}
& W(\alpha, t)=\int P\left(\alpha, \alpha^{\prime}, t\right) W\left(\alpha^{\prime}, 0\right) d^{2} \alpha^{\prime}, \\
& P\left(\alpha, \alpha^{\prime}, t\right) d \alpha=\operatorname{Prob}\left[\alpha \leq a(t)<\alpha+d \alpha \mid a(0)=\alpha^{\prime}\right],
\end{aligned}
$$

which, in differential form, is a genuine Fokker-Planck equation; that is, it guarantees that $W$ remains positive definite if it was so at the initial time. The Wigner function $W(\alpha, t)$ is just the average over $\beta_{j}$ of the $\mathcal{W}\left(\alpha,\left\{\beta_{j}\right\}, t\right)$ involved in Eq. (2). We stress, finally, that the Langevin equation (5) is formally identical to the
Heisenberg-Langevin equation which is obtained in the quantum formalism, starting from (1), after the elimination of the heat-bath degrees of freedom. In consequence, the Fokker-Planck equation associated with (9) is also the same as that obtained by the usual procedure starting from the quantum evolution equation of the density matrix transformed to the $W$ representation.

In order to study the solutions of Eq. (2), we shall choose the coupling

$$
f(t)=i \epsilon e^{-2 i \omega t},
$$

(where $\epsilon$ is real) which makes the Hamiltonian (1) an appropriate model for the light squeezing device through optical parametric oscillation below threshold, as we shall show later on. In this case, it is convenient to change from the complex stochastic process $a(t)$ to the real ones $x(t)$ and $y(t)$ through

$$
a(t)=[x(t)+i y(t)] e^{-i \omega t},
$$

and it is straightforward to get from (5) the FokkerPlanck equation

$$
\begin{aligned}
\frac{\partial W(x, y, t)}{\partial t}=\gamma[ & \frac{1}{4}(1+2 \bar{n})\left(\frac{\partial^{2} W}{\partial x^{2}}+\frac{\partial^{2} W}{\partial y^{2}}\right)+\frac{\partial}{\partial x}(x W) \\
& \left.+\frac{\partial}{\partial y}(y W)\right]-\epsilon\left(\frac{\partial}{\partial x}(x W)-\frac{\partial}{\partial y}(y W)\right) .
\end{aligned}
$$

Hence, by the well-known relation between the distribution functions $W$ and $P$ or $Q$, it is possible to obtain the generalized Fokker-Planck equation in the form

$$
\begin{aligned}
\frac{\partial P(x, y, t)}{\partial t}= & \gamma\left[\frac{1}{4}(1+s+2 \bar{n})\left[\frac{\partial^{2} P}{\partial x^{2}}+\frac{\partial^{2} P}{\partial y^{2}}\right]\right. \\
& \left.+\frac{\partial}{\partial x}(x P)+\frac{\partial}{\partial y}(y P)\right] \\
& -\epsilon\left[\frac{\partial}{\partial x}(x P)-\frac{\partial}{\partial Y}(y P)\right. \\
& \left.+\frac{s}{4}\left[\frac{\partial^{2} P}{\partial x^{2}}-\frac{\partial^{2} P}{\partial y^{2}}\right)\right] .
\end{aligned}
$$

where $s=0$ reproduces Eq. (12) for the $W$ representation and $s=-1$ (1) gives the $P(Q)$ representation. This is the same equation that would be obtained following the usual procedure, that is, starting with the evolution equation of the density matrix.

The superiority of the Wigner representation is clear in the comparison between (12) and (13); the former is a genuine Fokker-Planck equation, whereas the latter sometimes has an indefinite diffusion matrix. In the case of the $P$ representation, we obtain an unphysical "diffusion" at low temperature for all values of the coupling constant $\epsilon$, while the $Q$ representation gives such problems only in the case $\epsilon>2 \gamma$. We shall show below that the Hamiltonian (1) is appropriate for describing the squeezing of light only in the "below-threshold" case $\epsilon<\gamma$. It is generally considered, therefore, that for prac- 
tical purposes, it makes no difference whether we use the $W$ or $Q$ representations, though it is now widely understood that the $P$ representation is inadequate for the description of squeezed states.

However, we propose to show that the $Q$ representation is also unsatisfactory compared with $W$. The essential point is made by considering the Langevin equation associated with (13). It has the same formal appearances as (5), but instead of (7), the noise description is given by

$$
\begin{aligned}
& \langle L(t)\rangle=0, \quad\left\langle L(t) L\left(t^{\prime}\right)\right\rangle=-\epsilon s \delta\left(t-t^{\prime}\right) \\
& \left\langle L(t) L^{*}\left(t^{\prime}\right)\right\rangle=\gamma(1+s+2 \bar{n}) \delta\left(t-t^{\prime}\right) .
\end{aligned}
$$

This is rather unphysical in that it ascribes part of the noise to the coupling process rather than it being a property solely of the heat bath, as in (7). But the autocorrelations are different for the three representations, namely,

$$
\begin{aligned}
\langle x(t) x(t+\tau)\rangle= & \frac{1}{4} e^{-(\gamma-\epsilon)|\tau|} \\
& \times\left(\frac{\gamma}{\gamma-\epsilon}(1+2 \bar{n})+s\right), \\
\langle y(t) y(t+\tau)\rangle= & \frac{1}{4} e^{-(\gamma+\epsilon)|\tau|} \\
& \times\left(\frac{\gamma}{\gamma+\epsilon}(1+2 \bar{n})+s\right), \\
\langle x(t) y(t+\tau)\rangle= & 0,
\end{aligned}
$$

which would indicate that the three Fokker-Planck equations represent three different stochastic processes. We will show in a subsequent publication that the Wigner function $(s=0)$ gives the correct autocorrelation, and we shall discuss the possibility of experimentally distinguishing this result from the other two.

The procedure can be extended to nonlinear problems in some conditions. For the sake of clarity we shall consider as a specific example the optical parametric oscillator. ${ }^{5}$ It involves just two radiation modes with frequencies $\omega$ and $2 \omega$ with the Hamiltonian

$$
\begin{aligned}
H= & \omega \hat{a}_{1}^{\dagger} \widehat{a}_{1}+2 \omega \hat{a}_{2}^{\dagger} \widehat{a}_{2}+\sum_{j} \omega_{j} \hat{b}_{j}^{\dagger} \hat{b}_{j} \\
& +\sum_{l=1}^{2} \sum_{j} \chi_{j l}\left(\hat{a}_{l} \hat{b}_{j}^{\dagger}+\hat{a}_{l}^{\dagger} \hat{b}_{j}\right)+H_{\mathrm{NL}},
\end{aligned}
$$

where $H_{\mathrm{NL}}$ contains the nonlinear coupling and the pumping

$$
H_{\mathrm{NL}}=\frac{i K}{2}\left(\widehat{a}_{1}^{\dagger} \hat{a}_{2}-\widehat{a}_{1}^{2} \widehat{a}_{2}^{\dagger}\right)+i E\left(\widehat{a}_{2}^{\dagger} e^{-2 i \omega t}-\widehat{a}_{2} e^{2 i \omega t}\right)
$$

Hence it is straightforward to derive the Heisenberg equations of motion for $\widehat{a}_{1}$ and $\hat{a}_{2}$. These equations can be linearized if we assume that the fluctuation is small in comparison with the average value. ${ }^{6}$ In this case we can separate in $\hat{a}_{1}$ the deterministic ( $c$-number) part $c_{1}$ from the part $\hat{z}_{1}$, carrying the quantum fluctuations in the form

$$
\widehat{a}_{1}=\left(c_{1}+\widehat{z}_{1}\right) e^{-i \omega t}, \quad \hat{a}_{2}=\left(c_{2}+\hat{z}_{2}\right) e^{-2 i \omega t} .
$$

The neglect of quadratic terms in $\hat{z}_{1}, \hat{z}_{2}$ transforms the nonlinear Heisenberg equations into linear ones, and it is then possible to apply our previous results, that is, we may replace the Heisenberg operators $\hat{z}_{1}, \hat{z}_{2}$ by the stochastic processes $z_{1}, z_{2}$ and remove the bath terms to obtain the Langevin equations

$$
\begin{aligned}
& \hat{z}_{1}=A_{1}\left(c_{1}, c_{2}\right)+K c_{1}^{*} z_{2}+K c_{2} z_{1}^{*}-\gamma_{1} z_{1}+L_{1} e^{i \omega t}, \\
& \dot{z}_{2}=A_{2}\left(c_{1}, c_{2}\right)-K c_{1} z_{1}-\gamma_{2} z_{2}+L_{2} e^{2 i \omega t},
\end{aligned}
$$

where $\gamma_{1}, \gamma_{2}$ are inverse relaxation times and $L_{1}, L_{2}$ are complex independent white noises. The deterministic parts $A_{1}$ and $A_{2}$ (see below) have, by assumption, a greater order of magnitude than the fluctuations, represented by $z_{1}$ and $z_{2}$. Consequently, our linearization procedure will only be valid if they are removed from (19). That is, we must assume

$$
\begin{aligned}
& -\dot{c}_{1}+K c_{1}^{*} c_{2}-\gamma_{1} c_{1}=0, \\
& -\dot{c}_{2}-\frac{1}{2} K c_{1}^{2}+E-\gamma_{2} c_{2}=0 .
\end{aligned}
$$

These deterministic equations have two independent solutions, namely,

$$
\begin{aligned}
& c_{1}=0, \quad c_{2}=E / \gamma_{2}, \quad \text { valid for all } E \\
& c_{1}=\left[\frac{2 E}{K}-\frac{2 \gamma_{1} \gamma_{2}}{K^{2}}\right]^{1 / 2}, \quad c_{2}=\gamma_{1} / K, \\
& \text { if } E>\gamma_{1} \gamma_{2} / K .
\end{aligned}
$$

Substituted in (19), the first of these gives

$$
\begin{aligned}
& \dot{z}_{1}=\frac{K E}{\gamma_{2}} z_{1}^{*}-\gamma_{1} z_{1}+L_{1} e^{i \omega t}, \\
& \dot{z}_{2}=-\gamma_{2} z_{2}+L_{2} e^{2 i \omega t} .
\end{aligned}
$$

The damping matrix of the four-component real process $\left(z_{1}, z_{2}\right)$ is then

$\Gamma=\left(\begin{array}{cccc}-\gamma_{1}+\frac{K E}{\gamma_{2}} & 0 & 0 & 0 \\ 0 & -\gamma_{1}-\frac{K E}{\gamma_{2}} & 0 & 0 \\ 0 & 0 & -\gamma_{2} & 0 \\ 0 & 0 & 0 & -\gamma_{2}\end{array}\right)$,

which has all its eigenvalues negative provided that $E$ is less than the threshold value of $\gamma_{1} \gamma_{2} / K$. Below this threshold the approximation of discarding the quadratic terms in $z_{1}$ and $z_{2}$ will be justified provided the oscillations in $z_{2}$, produced by $L_{2}$, are much smaller than $c_{2}$. In that case the steady-state solution for the mode $a_{1}$ is the squeezed-vacuum state given by (15) with the replacements

$$
\epsilon=\frac{K E}{\gamma_{2}}, \quad \gamma=\gamma_{1}, \quad \bar{n}=\bar{n}_{1} .
$$

Note that the linearization has effectively decoupled $a_{1}$ from $a_{2}$; the sole effect of the pumping is to change the 
damping constants in the two quadrature components of $a_{1}$.

The mean-square fluctuations in these two components are

$$
\left\langle x^{2}\right\rangle=\frac{\gamma_{1}\left(1+2 \bar{n}_{1}\right)}{4\left(\gamma_{1}-K E / \gamma_{2}\right)}, \quad\left\langle y^{2}\right\rangle=\frac{\gamma_{1}\left(1+2 \bar{n}_{1}\right)}{4\left(\gamma_{1}+K E / \gamma_{2}\right)}
$$

The corresponding zero-point fluctuations are $\left\langle x^{2}\right\rangle=\left\langle y^{2}\right\rangle=\frac{1}{4}$, so that, for sufficiently low cavity temperature $\left(\bar{n}_{1} \rightarrow 0\right)$, the $y$ component is squeezed. Since the fluctuations are about the steady-state value $c_{1}=0$, this is a squeezed vacuum state, and the maximum possible squeezing is obtained as $E$ approaches threshold, that is,

$$
\left\langle y^{2}\right\rangle \rightarrow \frac{1}{8}, \quad \text { as } \bar{n}_{1} \rightarrow 0, \quad E \rightarrow \frac{\gamma_{1} \gamma_{2}}{K} .
$$

Note that the product of these mean squares is

$$
\left\langle x^{2}\right\rangle\left\langle y^{2}\right\rangle=\frac{1}{16}\left(1-\frac{K^{2} E^{2}}{\gamma_{1}^{2} \gamma_{2}^{2}}\right)^{-1}\left(1+2 \bar{n}_{1}\right)^{2} .
$$

Even at $T=0$ this is greater than the minimum value of $\frac{1}{16}$ required by the Heisenberg inequality. Thus the squeezed state is not a minimum uncertainty, or pure, quantum state; it cannot be represented by the application of a simple squeezing operator ${ }^{7}$ to the vacuum.

If on the other hand, for $E$ above threshold, we substitute (22) in (19), we find

$$
\begin{aligned}
& \dot{z}_{1}=\gamma_{1}\left(z_{1}^{*}-z_{1}\right)+\gamma^{\prime} z_{2}+L_{1} e^{i \omega t}, \\
& \dot{z}_{2}=-\gamma^{\prime} z_{1}-\gamma_{2} z_{2}+L_{2} e^{2 i \omega t},
\end{aligned}
$$

where

$$
\gamma^{\prime}=\left(2 E K-2 \gamma_{1} \gamma_{2}\right)^{1 / 2}
$$

In this case the damping matrix is

$$
\Gamma=\left(\begin{array}{cccc}
0 & 0 & \gamma^{\prime} & 0 \\
0 & -2 \gamma_{1} & 0 & \gamma^{\prime} \\
-\gamma^{\prime} & 0 & -\gamma_{2} & 0 \\
0 & \gamma^{\prime} & 0 & -\gamma_{2}
\end{array}\right),
$$

which has all its eigenvalues negative. Then again we conclude that the linearization is justified provided the oscillations produced by $L_{1}$ and $L_{2}$ are much smaller than $c_{1}$ and $c_{2}$. Assuming this is the case, and putting $z=x+i y$, we find, for the autocorrelations,

$$
\begin{aligned}
& \langle x(t) x(t+\tau)\rangle=\frac{1}{4 \gamma^{\prime 2} \gamma_{2}}\left(\left[\gamma_{1}\left(1+2 \bar{n}_{1}\right) \gamma_{2}^{2}+\gamma_{2}\left(1+2 \bar{n}_{2}\right) \gamma^{\prime 2}\right] \frac{\lambda_{2} e^{\lambda_{1}|\tau|}-\lambda_{1} e^{\lambda_{2}|\tau|}}{\lambda_{2}-\lambda_{1}}+\gamma_{1}\left(1+2 \bar{n}_{1}\right) \gamma^{\prime 2} \frac{\lambda_{2} e^{\lambda_{2}|\tau|}-\lambda_{1} e^{\lambda_{1}|\tau|}}{\lambda_{2}-\lambda_{1}}\right), \\
& \langle y(t) y(t+\tau)\rangle=\frac{1}{4 \gamma^{\prime 2}\left(\gamma_{2}+2 \gamma_{1}\right)} \mid\left[\gamma_{1}\left(1+2 \bar{n}_{1}\right) \gamma_{2}^{2}+\gamma_{2}\left(1+2 \bar{n}_{2}\right) \gamma^{\prime 2}\right] \frac{\lambda_{4} e^{\lambda_{3}|\tau|}-\lambda_{3} e^{\lambda_{4}|\tau|}}{\lambda_{4}-\lambda_{3}} \\
& \left.+\gamma_{1}\left(1+2 \bar{n}_{1}\right) \gamma^{\prime 2} \frac{\lambda_{4} e^{\lambda_{4}|\tau|}-\lambda_{3} e^{\lambda_{3}|\tau|}}{\lambda_{4}-\lambda_{3}}\right)
\end{aligned}
$$

where $\lambda_{i}(i=1-4)$ are the eigenvalues of $\Gamma$, that is,

$$
\begin{aligned}
& \lambda_{1}+\lambda_{2}=-\gamma_{2}, \\
& \lambda_{3}+\lambda_{4}=-\gamma_{2}-2 \gamma_{1}, \\
& \lambda_{1} \lambda_{2}=\lambda_{3} \lambda_{4}=\gamma^{\prime 2}
\end{aligned}
$$

and

$$
\begin{aligned}
& 1+2 \bar{n}_{1}=\operatorname{coth}\left(\omega / 2 k T_{1}\right), \\
& 1+2 \bar{n}_{2}=\operatorname{coth}\left(\omega / k T_{2}\right),
\end{aligned}
$$

with the temperatures $T_{1}$ and $T_{2}$ of the two cavities not necessarily equal. Again the $y$ component is squeezed, for if we put $\bar{n}_{1}$ and $\bar{n}_{2}$ zero, we obtain

$$
\left\langle y^{2}\right\rangle=\frac{\gamma_{1} \gamma_{2}^{2}+\gamma_{1} \gamma^{\prime 2}+\gamma_{2} \gamma^{\prime 2}}{4\left(\gamma_{2}+2 \gamma_{1}\right) \gamma^{\prime 2}}\left(\bar{n}_{1}=\bar{n}_{2}=0\right) .
$$

This is less than $\frac{1}{4}$ if $\gamma^{\prime 2}$ is greater than $\gamma_{2}^{2}$, that is, $E>\left(\gamma_{1} \gamma_{2}+\frac{1}{2} \gamma_{2}^{2}\right) / K$. As with the other linearized regime, it is a simple matter here also to demonstrate that we are dealing with a nonminimal squeezed state. Note that in this case, since $c_{1}$ is real and not zero, the fact that it is the $y$ component which is squeezed, means that the state may be described ${ }^{8}$ as phase-squeezed coherent.

The procedure we have described may be interpreted in one of two ways. On the one hand, we could say that we started with the Heiserberg-Langevin equations 
$\frac{d}{d t} \widehat{a}_{1}=-i\left(\omega-i \gamma_{1}\right) \widehat{a}_{1}+K \widehat{a}_{1}^{\dagger} \widehat{a}_{2}+\hat{L}_{1}$,

$\frac{d}{d t} \widehat{a}_{2}=-i\left(2 \omega-i \gamma_{2}\right) \hat{a}_{2}-\frac{1}{2} K \widehat{a}_{1}^{2}+E e^{-2 i \omega t}+\hat{L}_{2}$,

and then, after linearization, obtained the quantum master equation for the Wigner distribution. Such a procedure does indeed lead us to the approximate Langevin equations (23) or (27). However, a simpler, and apparently more heuristic, procedure would be to simply remove the hats in Eq. (38), that is, to write

$$
\begin{aligned}
& \dot{a}_{1}=-i\left(\omega-i \gamma_{1}\right) a_{1}+K a_{1}^{*} a_{2}+L_{1}, \\
& \dot{a}_{2}=-i\left(2 \omega-i \gamma_{2}\right) a_{2}-\frac{1}{2} K a_{1}^{2}+E e^{-2 i \omega t}+L_{2},
\end{aligned}
$$

and to regard this nonlinear Langevin equation as the stochastic differential equation for the process $\left(a_{1}, a_{2}\right)$. This may be linearized directly, that is, without passing through the master equation, and again we arrive at (23) or (27).

The treatment of the nonlinear Heisenberg-Langevin equation has so far proved to be an intractable problem and in any case the status of quantum Langevin equations is rather uncertain in the nonlinear regime. ${ }^{9}$ Its quantum master equation gives a Fokker-Planck equation which includes third derivatives, and so, by a general theorem of Pawula, ${ }^{10}$ a distribution which is initially positive will not, in general, stay positive. One could reasonably speculate as to whether this undesirable feature comes from the choice of model (with a single mode of the radiation field in each cavity), or whether it is really inevitable that the quantum world allows us only to treat "pseudoprobabilities" if we seek a phase-space description of it. In the meantime, we suggest that Eq. (39), even though it may be "derived" from (38) only in those regimes where linearization is valid, is worthy of further attention. Since it describes a genuine stochastic process, it will lead to a Fokker-Planck equation with positivedefinite fundamental solution. Furthermore, it will provide a smooth transition, across threshold, between the two linear regimes treated in the present article.

We acknowledge the financial support of Direccion General de Investigación Científica y Técnica (DGICYT) Project No. PB-87-0014 (Spain).
${ }^{1}$ M. Sargent, M. O. Scully, and W. E. Lamb, Laser Physics (Addison Wesley, Reading, MA, 1974), Chap. 16.

2J. Periňa, Quantum Statistics of Linear and Nonlinear Optical Phenomena (Reidel, Dordrecht, 1984), pp. 94-97.

${ }^{3}$ J. E. Moyal, Proc. Cambridge Philos. Soc. 45, 99 (1949).

${ }^{4}$ J. Periňa, Ref. 2, pp. 167-168.

5 J. Periňa, Ref. 2, p. 226.
${ }^{6}$ P. D. Drummond, K. J. McNeil, and D. F. Walls, Opt. Acta 28, 211 (1981).

${ }^{7}$ R. Loudon and P. L. Knight, J. Mod. Opt. 34, 709 (1987).

${ }^{8}$ R. E. Slusher and B. Yurke, Sci. Am. 258, 32 (1988).

${ }^{9}$ A. Caldeira and A. J. Leggett, Phys. A 121, 587 (1983).

${ }^{10}$ R. F. Pawula, IEEE Trans. Inf. Theory 13, 33 (1967). 